\title{
EDITORIAL
}

\section{The big three}

This month we publish a Review and an Expert Recommendation on multi-messenger astrophysics, a field that inherently involves big collaborations, big instruments and big data.

Last month, the Breakthrough Prize Foundation announced the 2020 Breakthrough Prize in Fundamental Physics and the New Horizons in Physics Prizes. The Event Horizon Telescope Collaboration bagged the Breakthrough Prize for "the first image of a supermassive black hole, taken by means of an Earth-sized alliance of telescopes"1 and one of the three New Horizons Prizes was awarded "for the development of novel techniques to extract fundamental physics from astronomical data" ${ }^{1}$. These prizes recognize three increasingly important components of physics research today: big collaborations, big instruments and big data.

In this issue, we focus on multi-messenger astrophysics, an emerging field that by its very nature involves large international collaborations working with big instruments in various locations in space and on the ground, producing vast amounts of data. Much like we use multi-sensory information to make sense of the world around us, multi-messenger astrophysics explores the Universe using the information carried by photons, gravitational waves, neutrinos and cosmic rays. Different types of instruments, such as telescopes, gravitational wave observatories, cosmic ray and neutrino detectors, serve as our eyes and ears. The information from the different messengers is brought together to be analysed and interpreted by large scientific collaborations, which can be thought of, in this anatomical comparison, as the central nervous system.

A Review by Péter Mészáros and colleagues covers the recent breakthroughs in multi-messenger astrophysics

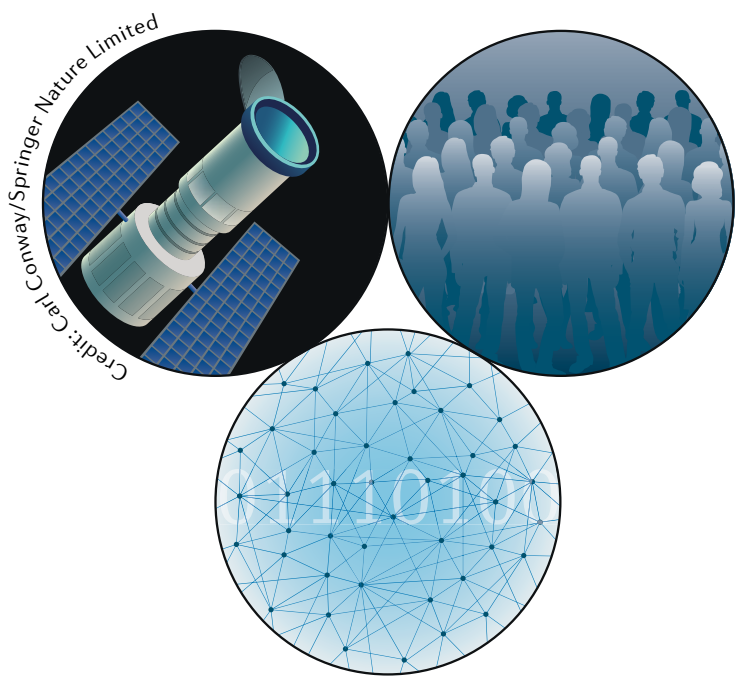

and the scientific questions that have subsequently been answered and the new questions that have emerged. Mészáros et al. discuss the challenges and opportunities in the field and survey the existing, upcoming and planned instruments.

One of the challenges in multi-messenger astrophysics lies in the analysis of the large amounts of observational data. Moreover, certain analyses, such as confirming the nature of a gravitational wave signal, need to be done in real time so that alerts can be sent to other instruments in time for possible detections of other messengers. Going back to the sensory analogy, this means that as soon as one hears a sound (of a gravitational wave event), one should turn their eyes (telescope) to the sky.

In an Expert Recommendation, Eliu Huerta and co-workers discuss the challenges in analysing real-time data from multi-messenger astrophysical events and the potential of deep learning algorithms in this area. They provide a series of recommendations on actions to be taken to enhance the potential for new discoveries in multi-messenger astrophysics. These include the development of data analysis algorithms and a cyber-infrastructure to support them, the management of real-time alerts and building the necessary community of experts - in other words, creating the artificial equivalent of the central nervous system to process the multi-messenger sensory information.

Expert Recommendation is a new article type introduced by Nature Reviews Physics to bring under the same umbrella articles previously published as Comments or Perspectives in the Nature journals. Expert Recommendations are peer-reviewed articles, authored by experts or panels of experts, that propose a course of action, best working practices or methodological guidelines. The recommendations can be the result of an analysis, on-going discussions in the community or a dedicated scientific meeting. Examples can be seen in this collection.

Nature Reviews Physics introduced Roadmaps and Expert Recommendations to support big collaborations, big instruments, big-data science. Much more can be done and we are keen to engage with the scientific community to explore how review-type articles can evolve to meet its changing needs.

\footnotetext{
Breakthrough Prize. Winners of the 2020 breakthrough prize in life sciences, fundamental physics and mathematics announced. Breakthrough Science https://breakthroughprize.org/News/54 (2019).
} 